\title{
Incidence of Periaortic Sustained Ventricular Tachycardias Long After Surgical Aortic Valve Replacements
}

\author{
Kentaro Goto ${ }^{1}$, Yuichi Ono ${ }^{1}$, Yuki Osaka ${ }^{1}$, Asami Suzuki ${ }^{1}$, Ken Kurihara ${ }^{1}$, Takeshi \\ Someya $^{1}$, Yoshihide Takahashi ${ }^{2}$, Kenichiro Otomo ${ }^{1}$, Masahiko Goya ${ }^{2}$, and Tetsuo Sasano ${ }^{2}$ \\ ${ }^{1}$ Ome Municipal General Hospital \\ ${ }^{2}$ Tokyo Medical and Dental University
}

June 12, 2020

\begin{abstract}
Objective: To investigate the incidence, risk factors and clinical characteristics of periaortic VTs after AVR. Background: The periaortic region is the origin of some ventricular tachycardias (VTs) after aortic valve replacement (AVR). However, the clinical characteristics of periaortic VTs after AVR are yet to be clarified. Methods: We retrospectively analyzed clinical courses of 109 patients who had undergone surgical AVR (SAVR) without other structural heart diseases between April 2009 and Jun 2019 and evaluated the incidence and characteristics of periaortic VTs after SAVR. Results: Three patients (2.8\%) developed periaortic VTs after SAVR. The average duration of onset was 12.3 \pm 6.6 years. All VTs arose from the inferior axis; they included both left and right bundle branch block configuration (LBBB and RBBB). Two patients underwent cardiac magnetic resonance imaging; late gadolinium enhancement (LGE) was observed in the mid-layer of the left ventricle basal anteroseptal wall in both cases. Patients with periaortic VTs had significantly wider interventricular septum, lower left ventricular ejection fraction (LVEF), larger LV diameter at systole, and higher positive rates of signal-averaged ECG and non-sustained VTs on Holter. On ablation, local fragmented potentials with low voltage zones were observed in accordance with the distribution of LGE. Multiple VTs originating from the periaortic region were provoked in the sessions. Conclusions: Periaortic VTs long after surgical AVR are not rare, and arrhythmia risk stratification, including that by signal averaged electrocardiogram (SAECG), Holter, and cardiac magnetic resonance imaging (MRI) should be considered.
\end{abstract}

\section{Incidence of Periaortic Sustained Ventricular Tachycardias Long After Surgical Aortic Valve Replacements}

Short Title: Periaortic ventricular tachycardias long after surgical aortic valve replacement originating from perivalvular scars.

Kentaro Goto, MD ${ }^{\mathrm{a}}$, Yuichi Ono, MD ${ }^{\mathrm{a}}$, Yuki Osaka, MD ${ }^{\mathrm{a}}$, Asami Suzuki, MD ${ }^{\mathrm{a}}$, Ken Kurihara MD ${ }^{\mathrm{a}}$, Takeshi Someya, $\mathrm{MD}^{\mathrm{b}}$, Yoshihide Takahashi, $\mathrm{MD}^{\mathrm{c}}$, Kenichiro Otomo, $\mathrm{MD}^{\mathrm{a}}$, Masahiko Goya, $\mathrm{MD}^{\mathrm{c}}$, and Tetsuo Sasano, $\mathrm{MD}^{\mathrm{c}}$.

${ }^{a}$ Division of Cardiovascular Medicine, Ome Municipal General Hospital,

4-16-5 Higashi Ome, Ome City, 198-0042, Tokyo, Japan.

${ }^{\mathrm{b}}$ Division of Cardiovascular Surgery, Ome Municipal General Hospital,

4-16-5 Higashi Ome, Ome City, 198-0042, Tokyo, Japan.

${ }^{\mathrm{c}}$ Department of Cardiovascular Medicine, Tokyo Medical and Dental University. 
1-5-45 Yushima, Bunkyo-ku, 113-8510, Tokyo, Japan.

\section{Address for correspondence:}

Kentaro Goto, MD,

Division of Cardiology, Ome Municipal General Hospital,

4-16-5 Higashi Ome, Ome City, 198-0042, Tokyo, Japan.

Tel 81-428-22-3191; Fax: 81-428-24-5126

E-mail address:ken-goto@umin.ac.jp

\section{Conflicts of Interest: None}

Word count: 4,182 words

\section{Structured Abstract}

Objective: To investigate the incidence, risk factors and clinical characteristics of periaortic VTs after AVR.

Background: The periaortic region is the origin of some ventricular tachycardias (VTs) after aortic valve replacement (AVR). However, the clinical characteristics of periaortic VTs after AVR are yet to be clarified.

Methods: We retrospectively analyzed clinical courses of 109 patients who had undergone surgical AVR (SAVR) without other structural heart diseases between April 2009 and Jun 2019 and evaluated the incidence and characteristics of periaortic VTs after SAVR.

Results: Three patients $(2.8 \%)$ developed periaortic VTs after SAVR. The average duration of onset was $12.3 \pm 6.6$ years. All VTs arose from the inferior axis; they included both left and right bundle branch block configuration (LBBB and RBBB). Two patients underwent cardiac magnetic resonance imaging; late gadolinium enhancement (LGE) was observed in the mid-layer of the left ventricle basal anteroseptal wall in both cases. Patients with periaortic VTs had significantly wider interventricular septum, lower left ventricular ejection fraction (LVEF), larger LV diameter at systole, and higher positive rates of signal-averaged ECG and non-sustained VTs on Holter. On ablation, local fragmented potentials with low voltage zones were observed in accordance with the distribution of LGE. Multiple VTs originating from the periaortic region were provoked in the sessions.

Conclusions: Periaortic VTs long after surgical AVR are not rare, and arrhythmia risk stratification, including that by signal averaged electrocardiogram (SAECG), Holter, and cardiac magnetic resonance imaging (MRI) should be considered.

(239 words)

Keywords: periaortic, ventricular tachycardia, aortic valve replacement, arrhythmia, long-term

\section{List of Abbreviations}

$\mathrm{AAD}=$ antiarrhythmic drugs

ACEIs = angiotensin-converting enzyme inhibitors

$\mathrm{AMC}=$ aortomitral continuity

ARBs $=$ angiotensin-II receptor blockers

$\mathrm{ASD}=$ atrial septal defect

$\mathrm{AVR}=$ aortic valve replacement

$\mathrm{CABG}=$ coronary artery bypass grafting

$\mathrm{DCM}=$ dilated cardiomyopathy 
$\mathrm{HCM}=$ hypertrophic cardiomyopathy

$\mathrm{INR}=$ international normalized ratio

$\mathrm{LGE}=$ late gadolinium enhancement

$\mathrm{MVP}=$ mitral valvuloplasty

$\mathrm{MVR}=$ mitral valve replacement

$\mathrm{PCI}=$ percutaneous coronary intervention

SAECG $=$ Signal averaged ECG

SAVR $=$ surgical AVR

$\mathrm{TTE}=$ transthoracic echocardiography

$\mathrm{VSD}=$ ventricular septal defect

$\mathrm{VT}=$ ventricular tachycardia

\section{Background}

The incidence of sudden death after cardiac valve replacement surgery has been reported to be $0.2-2.7 \%$ $(1,2)$. The main causes of sudden cardiac death include acute ischemic attack, worsening of congestive heart failure, and fatal arrhythmias (3-5).

As one of the fatal arrhythmias after aortic valve replacement (AVR), ventricular tachycardia (VT) arising from the periaortic region has been well-described in prior papers $(6,7)$. Among them, Liang JJ et al. summarized the electrophysiological characteristics of periaortic VTs after AVR in detail (6). In their report of 10 patients with nonischemic cardiomyopathies after AVR, VTs originated from the periaortic region in 7 (70\%). They also reported that periaortic scar detected by unipolar voltage mapping was observed in all 24 patients for whom detailed periaortic mapping was performed.

However, the incidence, time of onset, risk factors, and prognosis of periaortic VTs after AVR have not been investigated enough. This study aimed to investigate the clinical characteristics of periaortic VTs long after AVR.

\section{Methods}

\section{Study populations}

For this retrospective cohort study, we extracted and analyzed the medical data of 177 patients with past histories of SAVR at Ome Municipal General Hospital, Tokyo, Japan, between April 2009 and May 2019. Among them, 140 patients (79.1\%) underwent SAVR at our hospital, and the remaining 37 (20.9\%) presented to the outpatient clinic after SAVR that was performed in the other hospital.

Thirty-seven patients with follow-up $<1$ year were excluded. All patients underwent transthoracic echocardiography (TTE) and coronary angiography as a part of screening before SAVRs. We also excluded patients with structural heart diseases except for primary aortic valve diseases. The other structural heart diseases included a history of coronary artery disease, myocarditis, infiltrative heart diseases, including cardiac sarcoidosis and amyloidosis, congenital heart diseases, and other cardiomyopathies, such as hypertrophic cardiomyopathy (HCM) and dilated cardiomyopathy (DCM). Subsequently, the following patients were excluded: 29 with a history of ischemic heart disease, including old myocardial infarction and treatment history of percutaneous coronary intervention (PCI) or coronary artery bypass grafting $(\mathrm{CABG}) ; 1$ patient with atrial septal defect (ASD); and 1 patient with ventricular septal defect (VSD). Finally, the data of 109 patients who had undergone SAVR without diagnosed structural heart diseases other than primary aortic valve diseases were analyzed retrospectively.

Medications during follow-up periods 
As for medications for patients after SAVR, warfarin was used for anticoagulation in patients with mechanical valves at doses adjusted to achieve target international normalized ratio (INR) of 1.6-2.6 in older people ([?]70 years of age) and 2.0-3.0 in younger people ( $<70$ years of age). Antiplatelet drugs were used in patients with biological valves. The use of other medications, including beta-blockers, angiotensin-converting enzyme inhibitors (ACEIs), angiotensin-II receptor blockers (ARBs), and other anti-arrhythmia drugs was at the discretion of the primary outpatient doctors. Amiodarone was used for sporadic non-sustained ventricular tachycardia (NSVT) detected by Holter electrocardiogram (ECG).

Outpatient follow-up tests

After SAVR, all 109 patients underwent TTE and laboratory investigations at least once a year as a routine check. Signal averaged ECG (SAECG) and Holter ECG were performed if patients had suspected clinical symptoms of arrhythmias or they approved to undergo the tests to stratify the risk of arrhythmia. SAECG was considered positive if at least two of the following criteria were satisfied: filtered QRS duration $>114$ ms; terminal (last $40 \mathrm{~ms})$ QRS root means square (RMS) voltage $<20 \mu \mathrm{V}$; and low amplitude $(<40 \mu \mathrm{V})$ signal (LAS) duration $>38 \mathrm{~ms}$.

Endpoint

The primary endpoint was the occurrence of periaortic VT, which is defined as a sustained or incessant NSVT originating from the outflow tract (OT) detected with 12-lead ECG and not associated with ischemic heart disease.

\section{Cardiac MRI with Gadolinium (Gd) enhancement}

If patients presented with periaortic VTs, cardiac magnetic resonance imaging (MRI) with Gd enhancement (1.5-T scanner, Intera Nova dual, Philips, The Netherlands) was performed to detect periaortic scars if there were no contraindications. All MRI images were interpreted by radiology reviewers.

Electrophysiologic study, mapping, and ablation

Electrophysiologic study, mapping, and ablation were performed in patients with the periaortic VTs. Electroanatomic mapping (Carto3, Biosense Webster, USA, California, Irvine) was performed during sinus or paced rhythm to define the areas of low voltage and scarring (bipolar and unipolar voltage cutoffs: $<1.5$ and $<8.3 \mathrm{mV}$, respectively). The left ventricle (LV) was accessed mainly with the trans-septal or retrograde aortic approach in patients with a biological aortic valve. In those with a mechanical aortic valve, the valve was not crossed in a retrograde fashion. When sustained VTs were not present spontaneously, programmed electrical stimulation, burst pacing, and isoproterenol infusion $(1-10 \mu \mathrm{g} / \mathrm{min})$ were used to provoke ventricular arrhythmias. Ablation was performed using a non-irrigated 4.0-mm tip catheter (CARTO Navistar Catheter, Biosense Webster) with a power of $30-40 \mathrm{~W}$ and a temperature limit of $50{ }^{\circ} \mathrm{C}$. The ablation was deemed successful if there was immediate suppression and sustained VTs were absent after repeating the induction protocol.

\section{Statistical analysis}

Continuous variables are expressed as mean \pm standard deviation. Categorical variables are expressed as numbers (percentages). Continuous and categorical variables were compared using the Student'st -test and Fisher's exact test, respectively. All data were analyzed using JMP v12.0.0 (SAS Institute Inc.; Cary, NC, USA). $P$-values $<0.05$ were considered statistically significant.

Ethics and informed consent

This study was approved by our institutional review boards, and informed consent was obtained using an opt-out form.

\section{Results}

Baseline patient characteristics 
Of the 109 patients (65 men [59.6\%]; overall mean age: 73.4+-13.9 years), 60 (55.0\%) had mechanical valves and $49(45.0 \%)$ had biological valves. In addition to SAVR, $9(8.2 \%)$ patients had mitral valve replacement (MVR) and $3(2.8 \%)$ had mitral valvuloplasty (MVP). The primary aortic diseases were the following: aortic stenosis (AS) $(n=62 ; 56.9 \%)$, aortic regurgitation (AR) $(n=28 ; 25.7 \%)$, infective endocarditis (IE) $(n=18$; $16.5 \%)$, and rupture of sinus of Valsalva aneurysm $(n=1 ; 0.9 \%)$. The mean duration since AVR was 11.0+-8.5 years. Other basic patient characteristics are summarized in Table 1.

Patients with acute onset of periaortic VTs

Three $(2.8 \%)$ patients had episodes of sustained or incessant NSVT originating from OT (OTVT) that was detected using 12-lead ECG (Figure 1A/B/C). Additionally, one other patient (0.9\%) had sustained episodes of VT originating from the lateral portion of the LV, and two other patients (1.8\%) had sustained VTs of unknown origin. The clinical characteristics of the three patients with OTVTs are summarized in Table 2. All patients developed acute onset of chest discomfort related to the sustained or incessant NSVTs and were transported to the emergency department. Coronary angiography was performed to confirm that none of them had a worsening case of ischemic heart disease. Based on their clinical courses and 12-lead ECGs, they were diagnosed with periaortic VTs. The average duration between AVR and onset of periaortic VTs was 12.3+-6.7 years. Morphologically, all VTs had an inferior axis, and two patients and one patient, respectively, had right- and left-bundle branch block patterns. One VT patient with right-bundle branch block pattern had polymorphic configuration (Figure 1A/B/C). The mean cycle length of VTs was 310+-36.1 msec.

Among 109 patients after SAVR, four (3.7\%) without documented periaortic VTs were transported to the emergency department with sudden cardiopulmonary arrest (all patients with asystole on arrival). Six (5.5\%) had definite cardiac death related to worsening of congestive heart failure or ischemic attack, 18 (16.5\%) had non-cardiac death related to underlying diseases. Another 6 (5.5\%) died from unknown causes in the follow-up periods.

Comparison of patients with and without periaortic VT

We compared the clinical background data of the patients with periaortic VT (periaortic VT group; N=3) and those without periaortic VT (control group; N=106). (Table3) The type of valve (mechanical valve: $66.7 \%$ vs. $54.7 \%$, respectively; $P=1.0$ ), primary aortic valve disease (AS: $33.3 \%$ vs. $57.6 \%, P=0.6$; AR: $33.3 \%$ vs. $25.5 \%, P=1.0$; and IE: $33.3 \%$ vs. $16.0 \%, P=0.4$, respectively), and time since AVR (12.3+6.7 years vs. $10.9+-8.5$ years, respectively; $P=0.8$ ) were not significantly different between the groups. Other clinical factors, including age, male sex, body mass index (BMI), hypertension, dyslipidemia, diabetes mellitus, chronic kidney disease (CKD), and atrial fibrillation were also not significantly different between them. Similarly, the use of beta-blockers, ACEIs/ARBs, amiodarone, and anti-aldosterone drugs group 1 was not significantly different between the groups.

Echocardiography revealed that a peak aortic jet velocity (AV Vmax), Aortic Valve Area (AVA) and rate of paravalvular leakage (moderate-to-severe) were not different between the groups. (Table 4). However, TTE revealed that the periaortic VT group had significantly lower left ventricular ejection fraction (LVEF), wider interventricular septum (IVSd), and larger left ventricular internal dimension in systole (LVDs) than the control group $(56.0+-10.1$ vs. $65.9+-7.9 \%, P=0.04 ; 14.6+-3.4$ vs. $12.0+-2.0 \mathrm{~mm}, P=0.03$; and $36.0+-5.6$ vs. $27.7+-5.8 \mathrm{~mm}, P=0.02$; respectively).

Signal averaged ECG and Holter results

Overall, 34 patients, including three with periaortic VTs, underwent SAECG. Positive results were observed in all patients $(100.0 \%)$ in the periaortic VT group and 8/31 $(25.8 \%)$ patients in the control group. We also performed Holter monitoring in 45 patients, including three with periaortic VTs. Sporadic NSVTs were observed in all three patients with periaortic VT and 6/42 (14.3\%) patients in the control group. The positive rate was significantly higher in the periaortic VT group than that in the controls.

Cardiac MRI of patients with periaortic VT 
Two patients with periaortic VTs underwent cardiac MRI with Gd enhancement. One patient with periaortic VT (Case1) could not undergo cardiac MRI with Gd enhancement because of CKD. In two patients with periaortic VTs (Case2/3), the imaging revealed late Gd enhancement (LGE) in the basal mid-layer of the anteroseptal wall just below the prosthetic aortic valves in both cases. (Figure 2A/B/C).

Ablation for periaortic VTs

Two patients with periaortic VTs underwent catheter ablation. One patient (Case1) could not undergo ablation because of double mechanical valve replacement, which prevented access to the left ventricle.

In case2, clinical VT1 with LBBB and non-clinical VT2 with RBBB configuration were provoked. The mechanisms of the VTs were diagnosed with re-entry due to reproducible induction by programmed stimuli, and branch block re-entry was unlikely based on intracardiac electrocardiography. However, the precise circuits of the VT1/2 could not be analyzed because of their hemodynamic instability. On substrate mapping, local fragmented potentials with low voltage zones were observed in the RVOT septal position in accordance with the distribution of LGE on cardiac MRI. We obtained a good pace map for clinical VT1 in the septal RVOT below AV in the low voltage zone (Figure 3A/B/C) with pacing delay (Stimulus-QRS: 47.0ms) and for non-clinical VT2 in AMC near the site of aortic valve. Diastolic potentials during the VTs were not observed, respectively. We used radiofrequency in the areas of the septal RVOT and the AMC, which resulted in successful immediate suppression of each periaortic VT.

In case3, 3 types of VTs were provoked in the session. The mechanisms of VT1-3 were diagnosed with re-entry but precise circuits could not be analyzed in a manner similar to case 2 . We perfomed pace mapping and found that clinical VT1 originated from the left coronary cusp (LCC), VT2 was suspected from the epicardium side of the LV. VT3 was originated from RVOT and we confirmed the local fragmented potential and low voltage zone in RVOT septal side as same as case2. We used radiofrequency in the area of LCC and RVOT and succeeded to suppress the multiple VTs.

After ablation follow-up

Intravenous implantable cardioverter-defibrillator or subcutaneous-ICD were implanted for four cases with periaortic VTs as secondary prevention. The two patients who underwent VT ablation have not experienced a recurrence of periaortic VTs till now ( 1 and 4.3 years free from sustained VT events after the ablation, respectively). The other patient with double valve replacement developed recurrence of periaortic VTs 1.4 years after the first incidence, even while on amiodarone. In response to this, we increased the dose of beta-blockers, which successfully suppressed periaortic VTs till now.

\section{Discussion}

One of the main findings of this study was that $2.8 \%$ of patients developed acute onset of periaortic VT after SAVR retrospectively. Previous studies have reported the electrophysiological characteristics of VTs after AVR; however, the incidence rate of periaortic VTs after AVR remains unclear $(6,7)$. Although one of the major limitations of this study is that we could not distinguish between the periaortic VTs related to AVR and an idiopathic outflow/summit VT which shows outflow/septal LGE unrelated to the aortic valve (8), the incidence of such idiopathic OTVTs in the general population was reported to be 13.85 per 1000,000 patients $(0.001385 \%)$; therefore, the incidence of periaortic VTs after SAVR (2.8\%) in this retrospective cohort is apparently high (9). (Central illustration)

As for the timing of the periaortic VTs after AVR, a bimodal pattern has been reported; they either occur immediately postoperatively or years later $(6,7,10,11)$. The periaortic VTs in this study occurred $12.3+-6.7$ years after SAVR, which is in agreement with the results of previous studies. The high incidence of periaortic VTs after over 12 years raises a concern of causality.

The periaortic region could be an arrhythmic substrate for scar-related ventricular tachycardia, even with and without structural heart diseases $(8,12,13)$. Sabastiaan R.D. Piers et al. reported that two typical scar patterns including anteroseptal scar in nonischemic cardiomyopathy with contrast-enhanced MRI (13). 
From the high incidence of periaortic VT after SAVR and spatial distribution of the basal anteroseptal scars detected with cardiac MRI in this study, the periaortic scars appear to be influenced by chronic mechanical stimulation to the arrhythmic sensitive basal anteroseptal region by the artificial valve over a long time. In our study, patients in the periaortic VT group also had significantly wider IVSd than did those in the control group, which might reflect septal wall remodeling due to chronic stimulation by the prosthetic aortic valves. Therefore, we believe that if the periaortic scars occur in the septum after SAVR, they could lead to injury to the conduction system and, possibly, result in a bundle block re-entry VT, which was previously reported long after SAVR (7).

Another possible explanation for the periaortic scars may include hidden cardiomyopathies before SAVR. While all patients underwent screening tests before SAVR, hidden cardiomyopathies cannot be sufficiently ruled out completely. In fact, Castano et al. reported that $16 \%$ of patients with severe AS had hidden transthyretin cardiac amyloidosis (14). The presence of more clinical data on echocardiography, cardiac MRI, SAECG, and Holter earlier in the time course, including before AVR, would have been useful in proving when periaortic scar developed.

Another concern is how many of the other 105 patients without periaortic VTs also have abnormal substrate in the periaortic region. SAECG positive (7/30 [23\%]) and sporadic NSVTs were observed in 5/41 (23\%) patients without periaortic VTs; sustained OTVT or incessant NS-OTVT. They could be potential candidates for periaortic VTs, and risk stratification methods should be considered.

Regarding the prognosis of VTs after AVR, Liang JJ et al. demonstrated the safety and good outcomes of catheter ablation (6). In contrast, Nishimura et al. reported that sixty-five percent of reentrant circuits of periaortic VT with and without AVR had endocardial activation gaps within the tachycardia cycle length (three-dimensional circuitry), which were associated with higher rates of recurrence than two-dimensional complete circuits at 1 year ( $73 \%$ vs $37 \%$ ).In our study, we could not analyze the precise circuits of the periaortic VTs after AVR. However, because LGE, which could be the arrhythmic substrate of periaortic VTs, was present in the mid-layer of LV, the circuit could include three-dimensional circuitry and total elimination of the arrhythmic substrate with endocardial ablation alone appeared difficult. Soejima et al. also reported the importance of endo and epicardial mapping for ventricular tachycardia in patients with mechanical valves (15). We should analyze precise VT circuits after AVR and long-term prognosis for periaortic VTs after AVR.

Based on our results, we suggest that arrhythmic risk stratification for patients after SAVR should be considered. From our findings, wider IVSd, lower LVEF, larger LVDs, NSVTs on Holter monitoring, positive SAECG, and cardiac MRI should be considered prognostic factors in periaortic VTs after SAVR.

\section{Limitations}

This study included a small number of clinical events and patients for statistical analysis, and the design was a retrospective analysis at a single center. We could not distinguish between VTs related to AVR and idiopathic OTVTs precisely. More detailed analyses and comparisons between cardiac MRI and highdensity endo- and epi-mapping would be needed to understand the precise circuits of periaortic VTs after AVR. Large-scale, prospective studies are needed to validate our findings.

\section{Conclusions}

Periaortic VTs long after surgical AVR are not rare, and arrhythmia risk stratification, that by including SAECG, Holter, and cardiac MRI, should be considered.

\section{Acknowledgments: None}

Funding sources: This research did not receive any specific grant from funding agencies in the public, commercial, or not-for-profit sectors.

\section{References}


1. Torka MC, Salefsky BE, Hacker RW, et al. Intermediate clinical results after aortic valve replacement with the Carpentier-Edwards pericardial bioprosthesis. Ann Thorac Surg 1995; 60:S311-5. https://doi.org/10.1016/0003-4975(95)00294-u.

2. Fernandez J, Laub GW, Adkins MS et al. Early and late-phase events after valve replacement with the St. Jude Medical prosthesis in 1200 patients. J Thorac Cardiovasc Surg 1994; 107:394-406; discussion 406-7. https://doi.org/10.1016/S0022-5223(94)70084-2.

3. Rooney SJ, Moreno de la Santa P, Lewis PA, et al. Sudden death in a large prosthetic valve series based on a single prosthesis: experience with the medtronic Hall valve. J Heart Valve Dis 1994; 3:5-9.

4. Konishi Y, Matsuda K, Nishiwaki N, et al. Ventricular arrhythmias late after aortic and/or mitral valve replacement. Jpn Circ J 1985; 49:576-83. https://doi.org/10.1253/jcj.49.576

5. Burke AP, Farb A, Sessums L. Causes of sudden cardiac death in patients with replacement valves: an autopsy study. J Heart Valve Dis 1994; 3:10-6.

6. Liang JJ, Castro SA, Muser D, et al. Electrophysiologic substrate, safety, procedural approaches and outcomes of catheter ablation for ventricular tachycardia in patients after aortic valve replacement. JACC Clin Electrophysiol 2019; 5:28-38. https://doi.org/10.1016/j.jacep.2018.08.008.

7. Eckart RE, Hruczkowski TW, Tedrow UB, et al. Sustained ventricular tachycardia associated with corrective valve surgery. Circulation 2007; 116:200511. https://doi.org/10.1161/CIRCULATIONAHA.107.703157.

8. Nagashima K, Tedrow UB, Koplan BA, et al. Reentrant ventricular tachycardia originating from the periaortic region in the absence of overt structural heart disease. Circ Arrhythm Electrophysiol 2014; 7:99-106.https://doi.org/10.1161/CIRCEP.113.000870.

9. Sirichand S, Killu AM, Padmanabhan D, et al. Incidence of idiopathic ventricular arrhythmias: a population-based study. Circ Arrhythm Electrophysiol 2017; 10 . pii: e004662. https://doi.org/10.1161/CIRCEP.116.004662.

10. Mohammed N, Dandekar U, Osman F. Ventricular tachycardia following aortic valve replacement. Heart 2015; 101:793. https://doi.org/10.1136/heartjnl-2014-306971.

11. Zaker-Shahrak R, Altmann D, Sommer P, et al. Sustained monomorphic left ventricular outflow tract tachycardia early after aortic valve replacement. Cardiol J 2012; 19:3202.https://doi.org/10.5603/cj.2012.005\%.

12. Nishimura T, Beaser A, Aziz Z et al. Peri-Aortic Ventricular Tachycardia in Structural Heart Disease: Evidence of Localized Reentrant Mechanisms. Heart Rhythm. 2020 Apr 20. pii: S1547-5271(20)303507. doi: 10.1016/j.hrthm.2020.04.018.

13. Sebastiaan R D Piers, Qian Tao, Carine F B van Huls van Taxis, et al. Contrast-enhanced MRI-derived Scar Patterns and Associated Ventricular Tachycardias in Nonischemic Cardiomyopathy: Implications for the Ablation Strategy. Circ Arrhythm Electrophysiol. 2013 Oct;6(5):875-83. doi: 10.1161/CIRCEP.113.000537.

14. Castano A, Narotsky DL, Hamid N, et al. Unveiling transthyretin cardiac amyloidosis and its predictors among elderly patients with severe aortic stenosis undergoing transcatheter aortic valve replacement. Eur Heart J 2017; 38:2879-87. https://doi.org/10.1093/eurheartj/ehx350.

15. Soejima K, Nogami A, Sekiguchi Y et al. Epicardial catheter ablation of ventricular tachycardia in no entry left ventricle: mechanical aortic and mitral valves. Circ Arrhythm Electrophysiol. 2015; 8:381-9. 


\section{The Periaortic VT after SAVR}

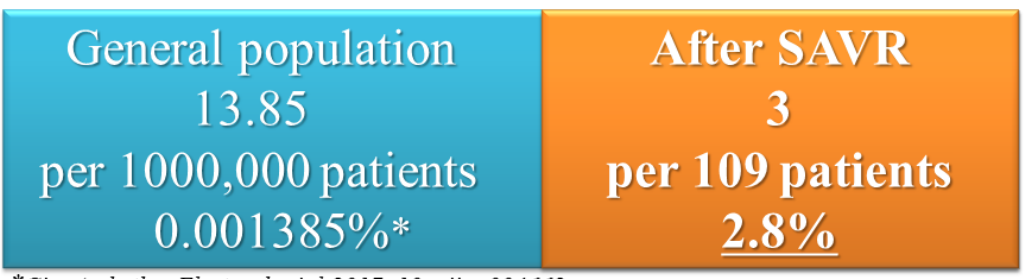

*Circ Arrhythm Electrophysiol 2017; 10. pii: e004662.

The average duration between AVR and

onset of periaortic VTs was $\underline{12.3 \pm 6.7}$ years.

\begin{tabular}{c|c|}
\hline -Morbidity- & - Clinical implication - \\
$\begin{array}{c}\text { Chronic mechanical stimulation } \\
\text { to the periaortic region by the } \\
\text { artificial valve for decades? }\end{array}$ & $\begin{array}{c}\text { Arrhythmic risk stratification should } \\
\text { be considered for patients after AVR } \\
\text { \# SAECG \# Holter \# cardiac MRI }\end{array}$ \\
\hline
\end{tabular}

\section{Central Illustration. Periaortic VT long after SAVR}

Figure Legend: Incidence rate of periaortic VT after SAVR was 3 per 109 patients (2.8\%). The average duration between AVR and the onset of periaortic VTs was $12.3 \pm 6.7$ years. The periaortic scars appear to originate from chronic mechanical stimulation to the arrhythmic sensitive periaortic region by the artificial valve for decades. Arrhythmic risk stratification for patients after AVR including that by SAECG, Holter, and cardiac MRI should be considered. Abbreviations: AVR, aortic valve replacement; ECG, electrocardiogram; MRI, magnetic resonance imaging; RBBB, right bundle branch block; SAECG, signal averaged electrocardiogram; SAVR, surgical aortic valve replacement; VT, ventricular tachycardia 


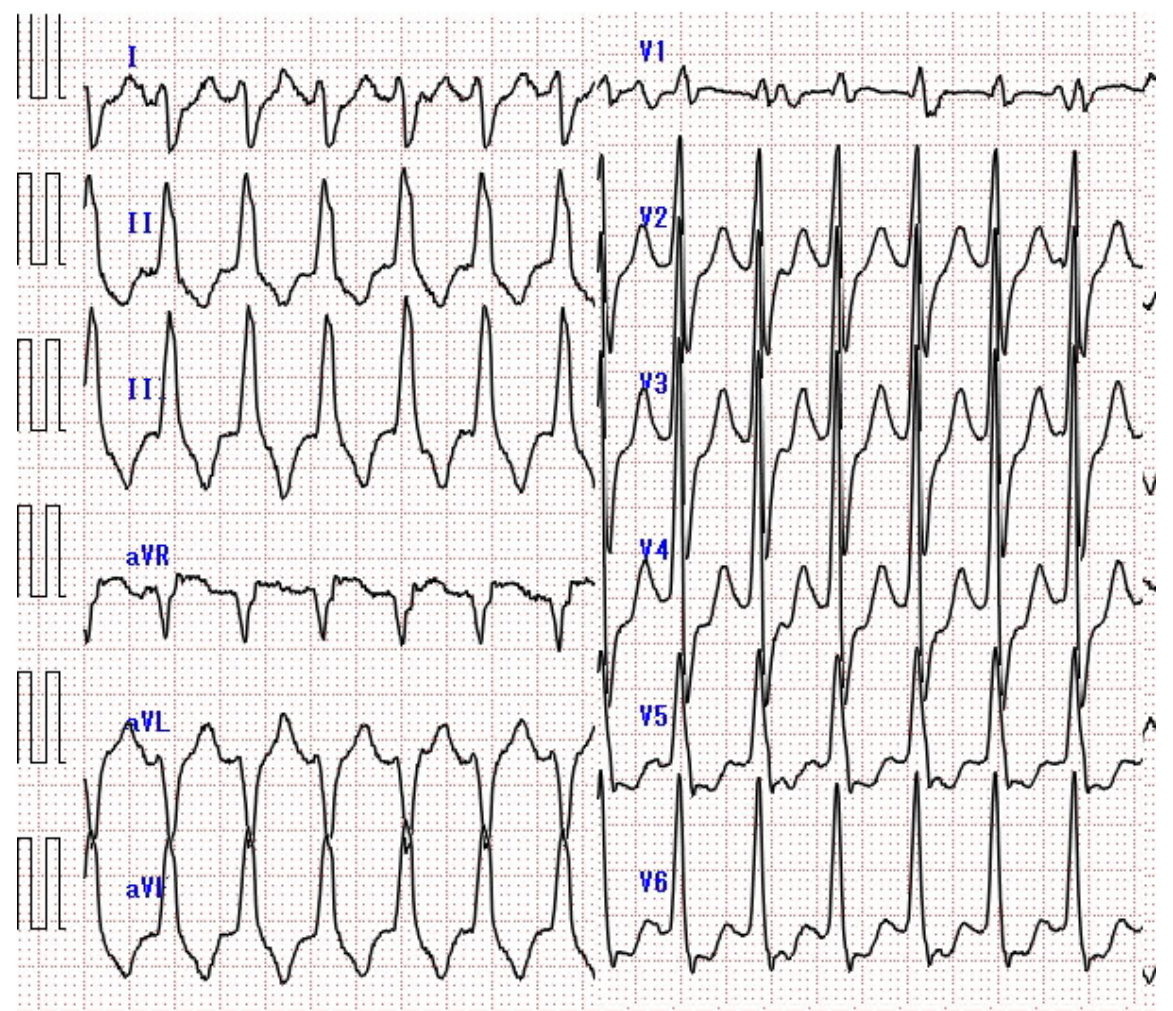

Figure 1A. 12-ECG of Periaortic VTs with RBBB configuration (Case1)

Figure Legend: ECG of monomorphic sustained Periaortic VT in Case 1 showed RBBB configuration, inferior axis, and a cycle length of $350 \mathrm{msec}$. (10.00 mm/mV. $25.0 \mathrm{~mm} / \mathrm{second})$

Abbreviations: ECG, electrocardiogram; RBBB, right bundle branch block; VT, ventricular tachycardia

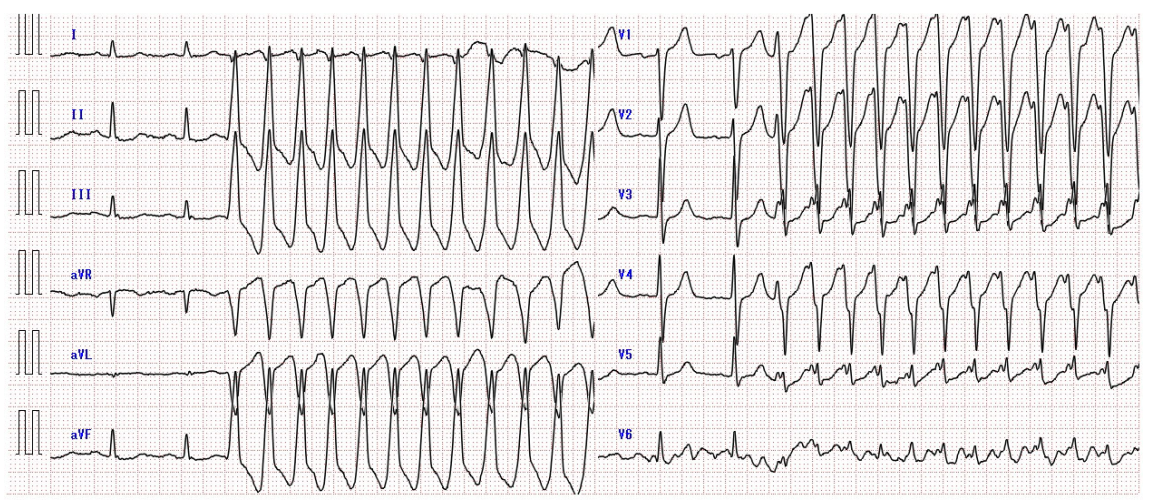

Figure 1B. 12-ECG of a Periaortic VT with LBBB configuration (Case2)

Figure Legend: ECG of monomorphic sustained Periaortic VT in Case 2 showed LBBB configuration, inferior axis, and a cycle length of $300 \mathrm{msec}$. (10.00 mm/mV. $25.0 \mathrm{~mm} / \mathrm{second})$

Abbreviations: ECG, electrocardiogram; LBBB, left bundle branch block; VT, ventricular tachycardia 


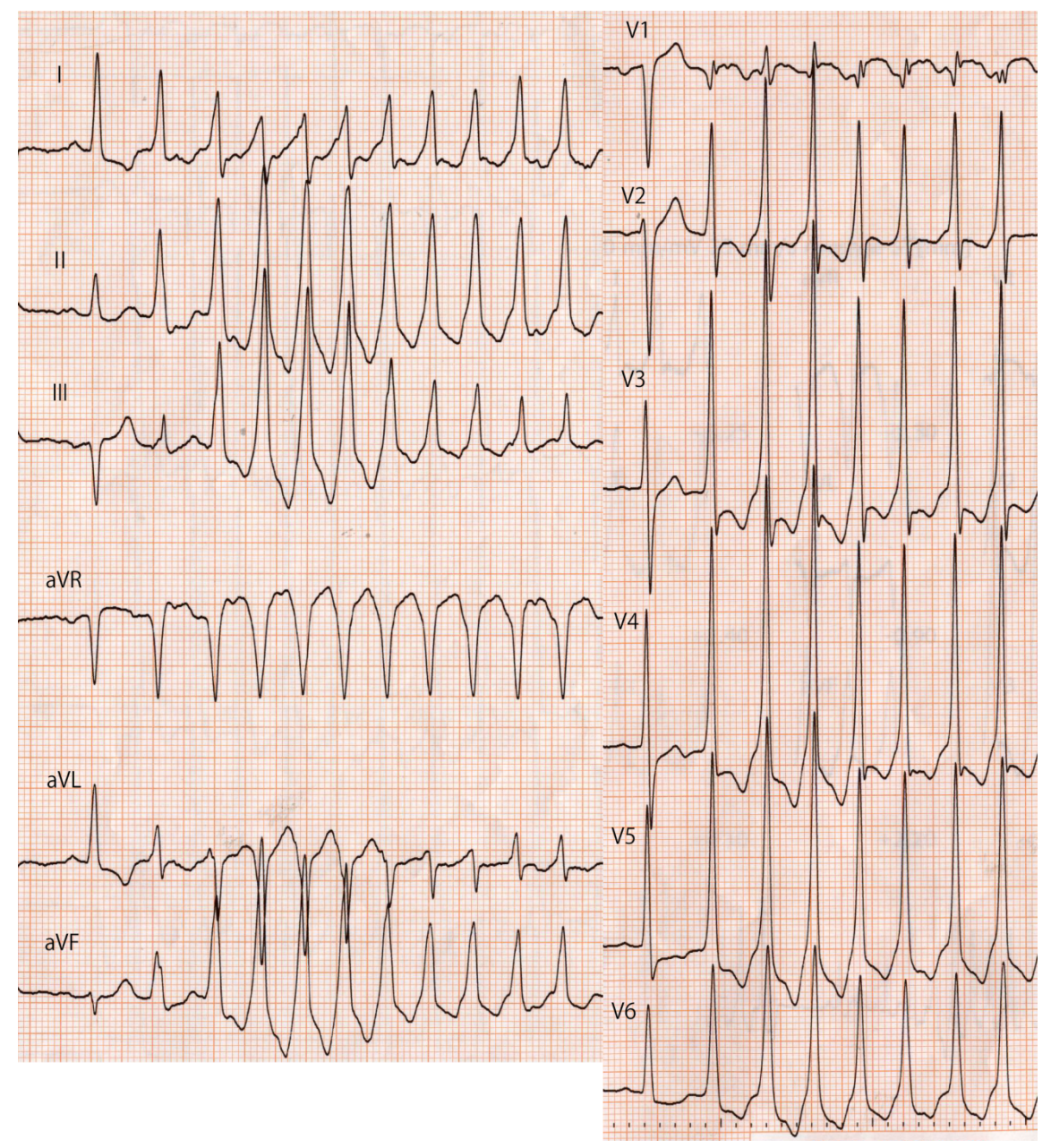

Figure 1C. 12-ECG of a Periaortic VT with RBBB configuration (Case3)

Figure Legend: ECG of polymorphic incessant non-sustained Periaortic VT in Case 3 showed LBBB configuration, inferior axis, and a cycle length of $280 \mathrm{msec}$. $(10.00 \mathrm{~mm} / \mathrm{mV} .25 .0 \mathrm{~mm} / \mathrm{second}$ ) Abbreviations: ECG, electrocardiogram; LBBB, left bundle branch block; VT, ventricular tachycardia 


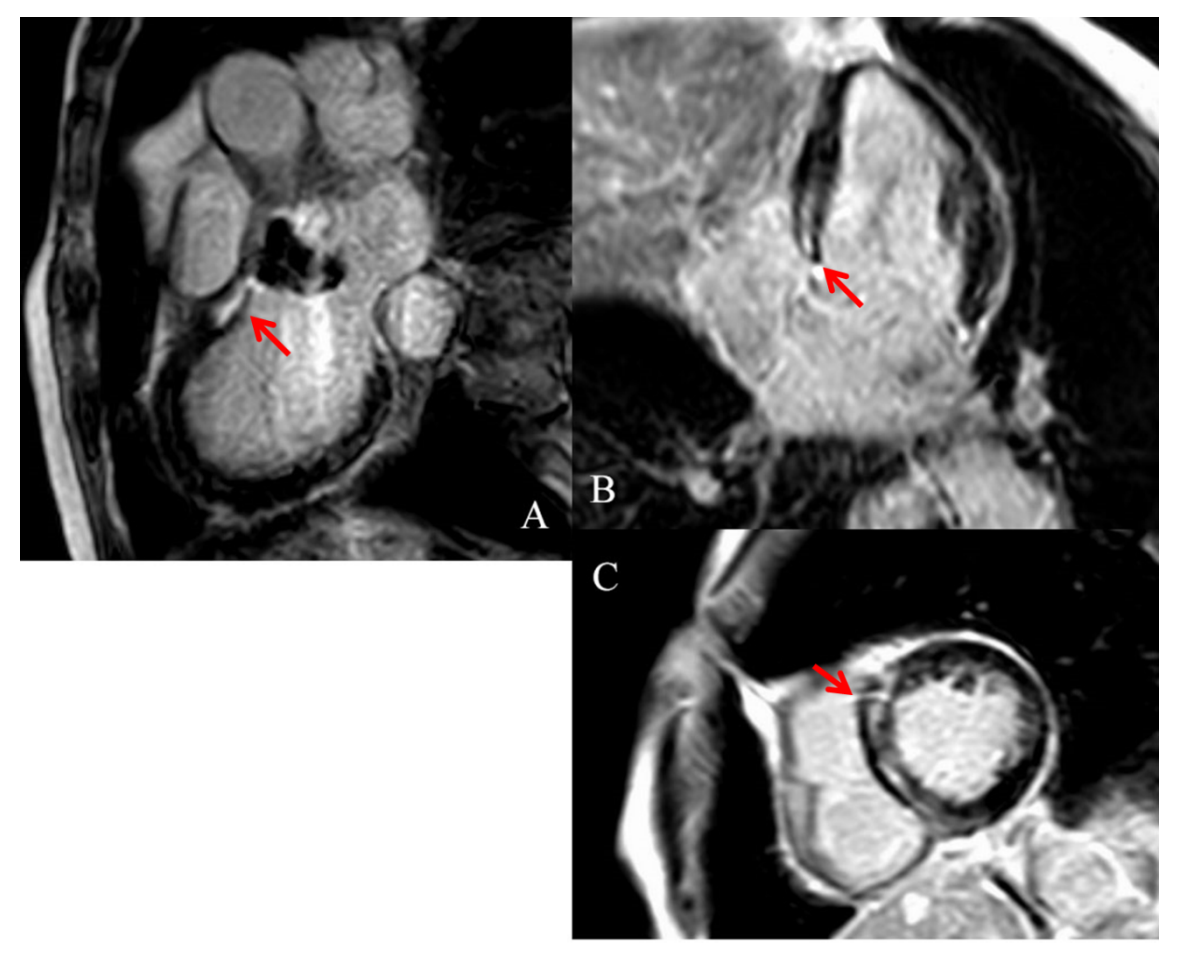

Figure 2A, B, C. LGE in a Patient with a Periaortic VT (Case 2)

(A: sagittal view, B: horizontal view, C: oblique section)

Figure Legend: LGE was observed in the mid-layer of basal RV/LV antero-septum just below the aortic valve. (A/B/C: Red allow)

Abbreviations: LGE, late gadolinium enhancement; VT, ventricular tachycardia 


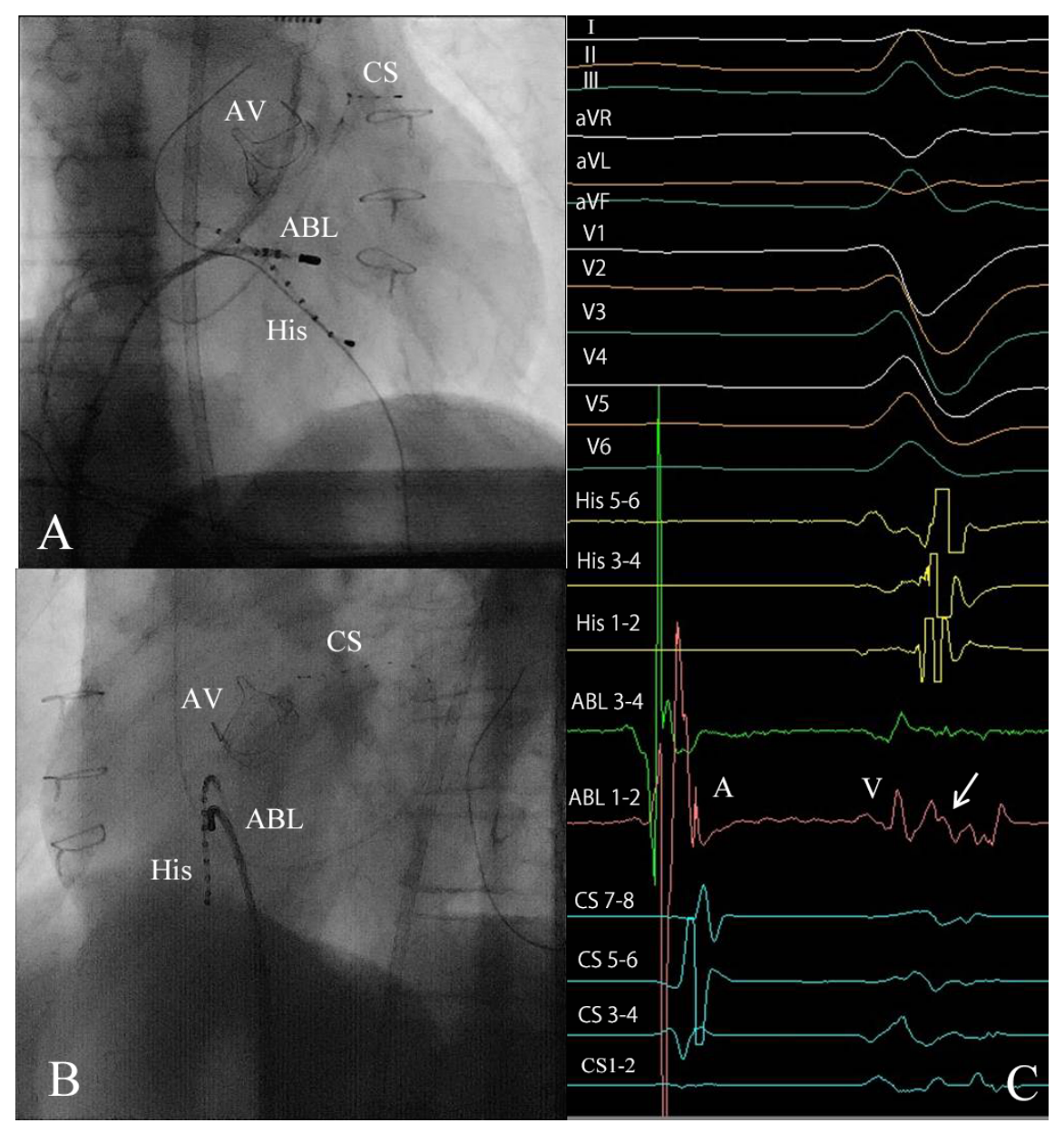

Figure 3A, B, C. Local abnormal electrocardiogram of Periaortc VT from RVOT (Case 2)

Figure Legend: Ablation catheter was positioned in the RV/LV septum below the artificial aortic valve from the right ventricular outflow tract (A/B). Intracardiac electrocardiogram revealed fragmented potentials in low voltage zones (C: white allow), and we also got a good pace map for the target periaortic VT in the same position.

Abbreviations: ABL, ablation; CS, coronary sinus; LBBB, left bundle branch block; LGE, late gadolinium enhancement; LV, left ventricle; RV, right ventricle; RVOT, right ventricular outflow tract; SR, sinus rhythm; VT, ventricular tachycardia

\section{Tables}

Table 1. Baseline Characteristics of Patients Underwent SAVR without Other Structural Heart Diseases $(\mathrm{N}=109)$

\begin{tabular}{ll}
\hline Age (years) & $73.4 \pm 13.9$ \\
\hline Male & $65(59)$ \\
BMI $\left(\mathrm{kg} / \mathrm{cm}^{2}\right)$ & $22.0 \pm 4.2$ \\
Aortic valve type & $60(55)$ \\
Mechanical valve & $49(45)$ \\
Biological valve &
\end{tabular}




\begin{tabular}{ll}
\hline Age (years) & $73.4 \pm 13.9$ \\
\hline Mitral valve operation & $12(10)$ \\
Primary aortic valve diseases & \\
AS & $62(56)$ \\
AR & $28(25)$ \\
IE & $18(16)$ \\
Others & $1(0)$ \\
Period after AVR (years) & $11.0 \pm 8.5$ \\
HT & $67(61)$ \\
DL & $53(48)$ \\
DM & $26(23)$ \\
CKD & $55(50)$ \\
Hemodialysis & $5(4)$ \\
Old cerebral infarction & $11(10)$ \\
Paroxysmal atrial fibrillation & $9(8)$ \\
Persistent atrial fibrillation & $16(14)$ \\
Atrial tachycardia & $1(0)$ \\
Medication & \\
Beta blocker & $62(56)$ \\
ACEI/ARB & $40(36)$ \\
Aldosterone antagonist & $9(8)$ \\
Amiodarone & $7(6)$ \\
Antiarrhythmic drug group 1 & $3(2)$ \\
\hline
\end{tabular}

Abbreviations: ACEI/ARB, angiotensin-converting enzyme inhibitors/angiotensin II receptor blockers; AR, Aortic regurgitation; AS, Aortic stenosis; AVR, aortic valve replacement; BMI, body mass index; CKD, Chronic kidney disease; CRT-D, Cardiac resynchronization therapy-defibrillator; DM, Diabetes mellitus; DL, Dyslipidemia; HT, Hypertension; ICD, implantable cardioverter defibrillator; IE, Infective endocarditis; Others, Rupture of sinus of Valsalva aneurysm

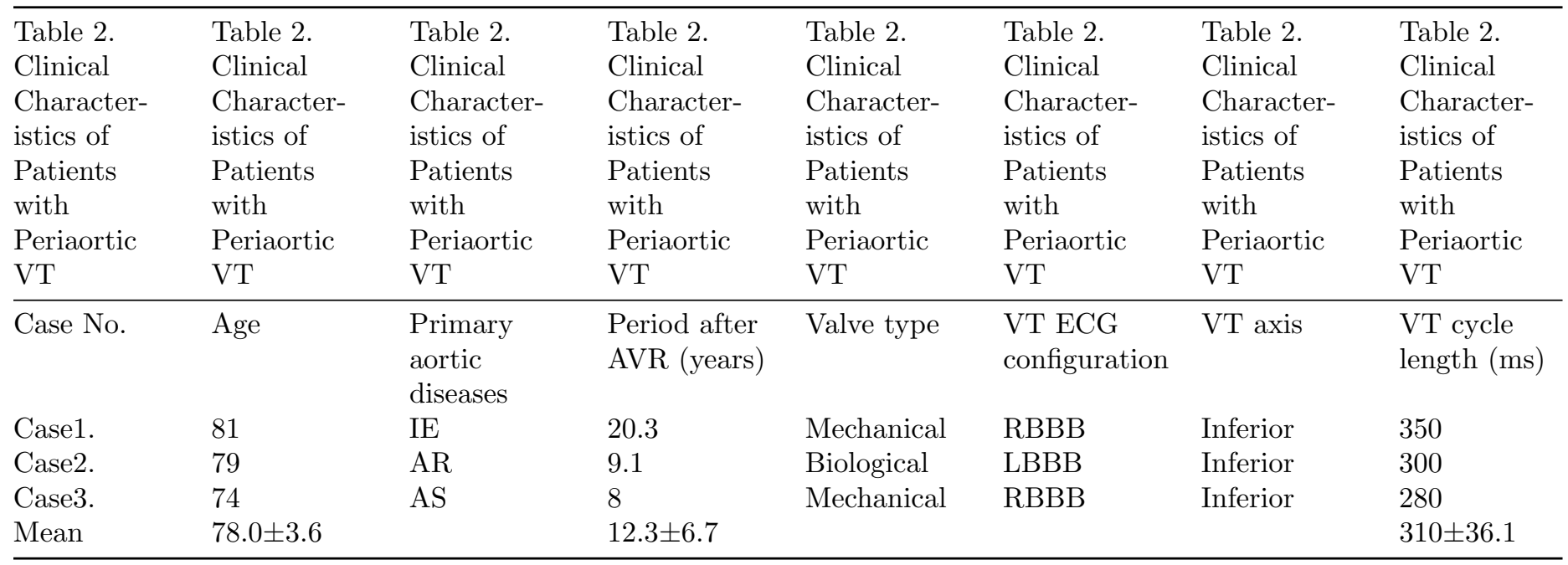

Abbreviations: AR, Aortic regurgitation; AS, Aortic stenosis; AVR, aortic valve replacement; ECG, electrocardiogram; IE, Infective endocarditis; LBBB, left bundle branch block; RBBB, right bundle branch block; VT, ventricular tachycardia 


\begin{tabular}{|c|c|c|c|}
\hline $\begin{array}{l}\text { Table 3. Comparison } \\
\text { between Patients with } \\
\text { Periaortic VT and } \\
\text { Controls }\end{array}$ & $\begin{array}{l}\text { Table 3. Comparison } \\
\text { between Patients with } \\
\text { Periaortic VT and } \\
\text { Controls }\end{array}$ & $\begin{array}{l}\text { Table 3. Comparison } \\
\text { between Patients with } \\
\text { Periaortic VT and } \\
\text { Controls }\end{array}$ & $\begin{array}{l}\text { Table } 3 \text {. Comparison } \\
\text { between Patients with } \\
\text { Periaortic VT and } \\
\text { Controls }\end{array}$ \\
\hline & $\begin{array}{l}\text { Periaortic VT group } \\
(\mathrm{N}=3)\end{array}$ & Control group $(\mathrm{N}=106)$ & $P$-value \\
\hline $\begin{array}{l}\text { Type of aortic valve } \\
\text { (mechanical valve) } \\
\text { Primary aortic valve } \\
\text { diseases }\end{array}$ & $2(66)$ & $58(54)$ & 1.0 \\
\hline $\mathrm{AS}$ & $1(33)$ & $61(57)$ & 0.6 \\
\hline $\mathrm{AR}$ & $1(33)$ & $27(25)$ & 1.0 \\
\hline $\mathrm{IE}$ & $1(33)$ & $17(16)$ & 0.4 \\
\hline $\begin{array}{l}\text { Periods after AVR } \\
\text { (years) }\end{array}$ & $12.3 \pm 6.7$ & $10.9 \pm 8.5$ & 0.8 \\
\hline Male & $3(100)$ & $62(58)$ & 0.3 \\
\hline Age (years) & $78.0 \pm 3.6$ & $73.3 \pm 14.1$ & 0.6 \\
\hline BMI $\left(\mathrm{kg} / \mathrm{cm}^{2}\right)$ & $23.8 \pm 2.2$ & $22.2 \pm 4.2$ & 0.5 \\
\hline HT & $2(67)$ & $65(61)$ & 1.0 \\
\hline DL & $2(67)$ & $51(48)$ & 0.6 \\
\hline DM & $0(0)$ & $26(24)$ & 1.0 \\
\hline CKD & $2(67)$ & $53(50)$ & 1.0 \\
\hline $\begin{array}{l}\text { Hemodialysis } \\
\text { Medication }\end{array}$ & $0(0 \%)$ & $5(5 \%)$ & 1.0 \\
\hline Beta blocker & $2(67)$ & $60(57)$ & 1.0 \\
\hline ACEI/ARB & $1(33)$ & $39(37)$ & 1.0 \\
\hline Aldsterone antagonist & $0(0)$ & $9(8)$ & 1.0 \\
\hline Amiodarone & 1(33) & $6(6)$ & 0.2 \\
\hline $\begin{array}{l}\text { Antiarrhythmic drug } \\
\text { group } 1\end{array}$ & $0(0)$ & $3(3)$ & 1.0 \\
\hline $\begin{array}{l}\text { Paroxysmal atrial } \\
\text { fibirillation }\end{array}$ & $0(0)$ & $9(8)$ & 1.0 \\
\hline $\begin{array}{l}\text { Persistent atrial } \\
\text { fibrillation }\end{array}$ & $0(0)$ & $16(15)$ & 1.0 \\
\hline
\end{tabular}

Abbreviations: ACEI/ARB, angiotensin-converting enzyme inhibitors/angiotensin II receptor blockers; AR, aortic regurgitation; AS, aortic stenosis; AVR, aortic valve replacement; BMI, body mass index; CKD, Chronic kidney disease; DM, Diabetes mellitus; DL, Dyslipidemia; HT, Hypertension; ICD/CRT-D, implantable cardioverter-defibrillator/cardiac resynchronization therapy defibrillator; IE, infective endocarditis; VT, ventricular tachycardia

\begin{tabular}{|c|c|c|c|}
\hline $\begin{array}{l}\text { Table 4. Comparison of } \\
\text { Echocardiography }\end{array}$ & $\begin{array}{l}\text { Table 4. Comparison of } \\
\text { Echocardiography }\end{array}$ & $\begin{array}{l}\text { Table 4. Comparison of } \\
\text { Echocardiography }\end{array}$ & $\begin{array}{l}\text { Table 4. Comparison of } \\
\text { Echocardiography }\end{array}$ \\
\hline Findings between & Findings between & Findings between & Findings between \\
\hline Patients with & Patients with & Patients with & Patients with \\
\hline Periaortic VT and & Periaortic VT and & Periaortic VT and & Periaortic VT and \\
\hline \multirow[t]{2}{*}{ Controls } & Controls & Controls & Controls \\
\hline & $\begin{array}{l}\text { Periaortic VT group } \\
(\mathrm{N}=3)\end{array}$ & Control group $(\mathrm{N}=106)$ & $P$-value \\
\hline AV $\operatorname{Vmax}(\mathrm{m} / \mathrm{sec})$ & $3.05 \pm 0.05$ & $2.69 \pm 0.67$ & 0.3 \\
\hline
\end{tabular}




\begin{tabular}{llll}
\hline Table 4. Comparison of & Table 4. Comparison of & Table 4. Comparison of & Table 4. Comparison of \\
Echocardiography & Echocardiography & Echocardiography & Echocardiography \\
Findings between & Findings between & Findings between & Findings between \\
Patients with & Patients with & Patients with & Patients with \\
Periaortic VT and & Periaortic VT and & Periaortic VT and & Periaortic VT and \\
Controls & Controls & Controls & Controls \\
\hline AVA $\left(\mathrm{cm}^{2}\right)$ & $1.58 \pm 0.09$ & $1.64 \pm 0.41$ & 0.8 \\
LVEF $(\%)$ & $56.0 \pm 10.1$ & $65.9 \pm 7.9$ & 0.04 \\
LVDd $(\mathrm{mm})$ & $50.7 \pm 5.1$ & $43.9 \pm 6.4$ & 0.08 \\
LVDs $(\mathrm{mm})$ & $36.0 \pm 5.6$ & $27.7 \pm 5.8$ & 0.02 \\
IVSd $(\mathrm{mm})$ & $14.6 \pm 3.5$ & $12.0 \pm 2.0$ & 0.03 \\
LVPWd $(\mathrm{mm})$ & $12.6 \pm 1.1$ & $11.3 \pm 1.5$ & 0.2 \\
Paravalvular leak & $1(33)$ & $6(6)$ & 0.2 \\
\hline
\end{tabular}

Abbreviations: AV, aortic valve; AVA, aortic valve area; IVSd, interventricular septum; LVDd, left ventricular diastolic dimension; LVDs, left ventricular end-systolic dimension 